\title{
The feasibility of a mandibular movement test as a screening tool for polysomnography candidates
}

\author{
To the Editor:
}

Sleep apnoea syndrome (SAS) is a common sleep disorder with a prevalence ranging from 5.9\% to $79.2 \%$ in the European general population over 40 years of age, depending on the clinical symptoms and apnoea hypopnoea scoring criteria used [1]. Despite its frequency, the fact that it is a significant risk factor for many common diseases, and a recent meta-analysis demonstrating that available treatments are effective [2], SAS remains underdiagnosed.

Indeed, patients with sleep apnoea presenting with concomitant cardiovascular diseases often have no typical presentation pointing towards obstructive SAS [3]. In various locations around the world, suspected SAS patients may find that accessing diagnostic examinations can be challenging. The average waiting time for a sleep study can vary from 2 to 48 months in industrialised countries [4]. Nevertheless, in-lab full-night polysomnography (PSG) remains the 'gold-standard' for the diagnosis of SAS, despite the fact that it is time consuming, expensive and often not widely available [5]. In certain countries, PSG is obligatory in the diagnostic work-up for SAS. Thus, targeting the patient population that is most likely to benefit from PSG would help to optimise the limited resources at hand.

Recent studies have suggested that mandibular movements (MM) can be considered a surrogate for the measurement of respiratory effort during sleep and can estimate total sleep time (eTST), as well as sleep fragmentation. Thus, MM can be used to identify sleep-disordered breathing $[6,7]$. In their study, MARTINOT et al. [7] compared the respiratory disturbance index measured by MM (MM-RDI) and the usual respiratory disturbance index measured by PSG (PSG-RDI). They reported that the MM-RDI is highly concordant with the PSG-RDI: in patients with a moderate-to-high pre-test probability for obstructive SAS, a MM-RDI $>13.5$ per h has a false positive rate of $4.72-18.49 \%$ (95\% CI) when detecting a PSG-RDI $\geqslant 15$ per $h$, and a false negative rate of $0.0-16.82 \%(95 \% \mathrm{CI})$.

Our objective was therefore to assess the feasibility of use of the MM-RDI as a screening tool in an ambulatory setting for PSG candidates. In order to address this question, we report herein the results of a preliminary study based on the retrospective analysis of a large, anonymous patient database in Belgium (clinicaltrials.gov identifier number NCT03129984).

From 2015-2016, data were collected for 4231 Belgian patients (figure 1), on whom automated MM tracking was performed (as prescribed by their practitioner), by the attachment of a type IV sleep monitor that included a MM magnetic sensor (Brizzy, Nomics, Liege, Belgium), under ambulatory conditions. In order to determine whether or not a given patient required PSG, we used the previously reported MM-RDI cut-off at $>13.5$ per $\mathrm{h}$ and a minimal eTST of over $4 \mathrm{~h}$, if MM-RDI $\leqslant 13.5$ per $\mathrm{h}$ [7].

The prescriber was a cardiologist in $80.9 \%$ of the cases (the cardiologist prescribers (CP) group in figure 1), and in $19.1 \%$ of the cases (the non-cardiologist prescribers (NCP) group), the prescribers comprised other types of practitioners (a general practitioner in $6.59 \%$, an otorhinolaryngologist in $4.62 \%$, a pulmonologist in $3.89 \%$, an orthodontist in $3.59 \%$, or another type of practitioner in $0.52 \%$ of cases). Out of 4231 tests, 165 (3.9\%) were unusable because of a major technical failure (damaged sensor, incorrectly positioned sensor, battery not recharged, too-short monitoring time or other technical/electronic problems). Of the 4066 remaining patients, only $9.4 \%$ of the analysable population had an estimated total sleep time below $4 \mathrm{~h}$.

@ERSpublications

Mandibular movement tests have demonstrated potential as screening tools for polysomnography candidates http://ow.ly/iu2430fZk2u

Cite this article as: Rotty M-C, Mallet J-P, Borel J-C, et al. The feasibility of a mandibular movement test as a screening tool for polysomnography candidates. Eur Respir J 2017; 50: 1701076 [https://doi.org/ 10.1183/13993003.01076-2017]. 


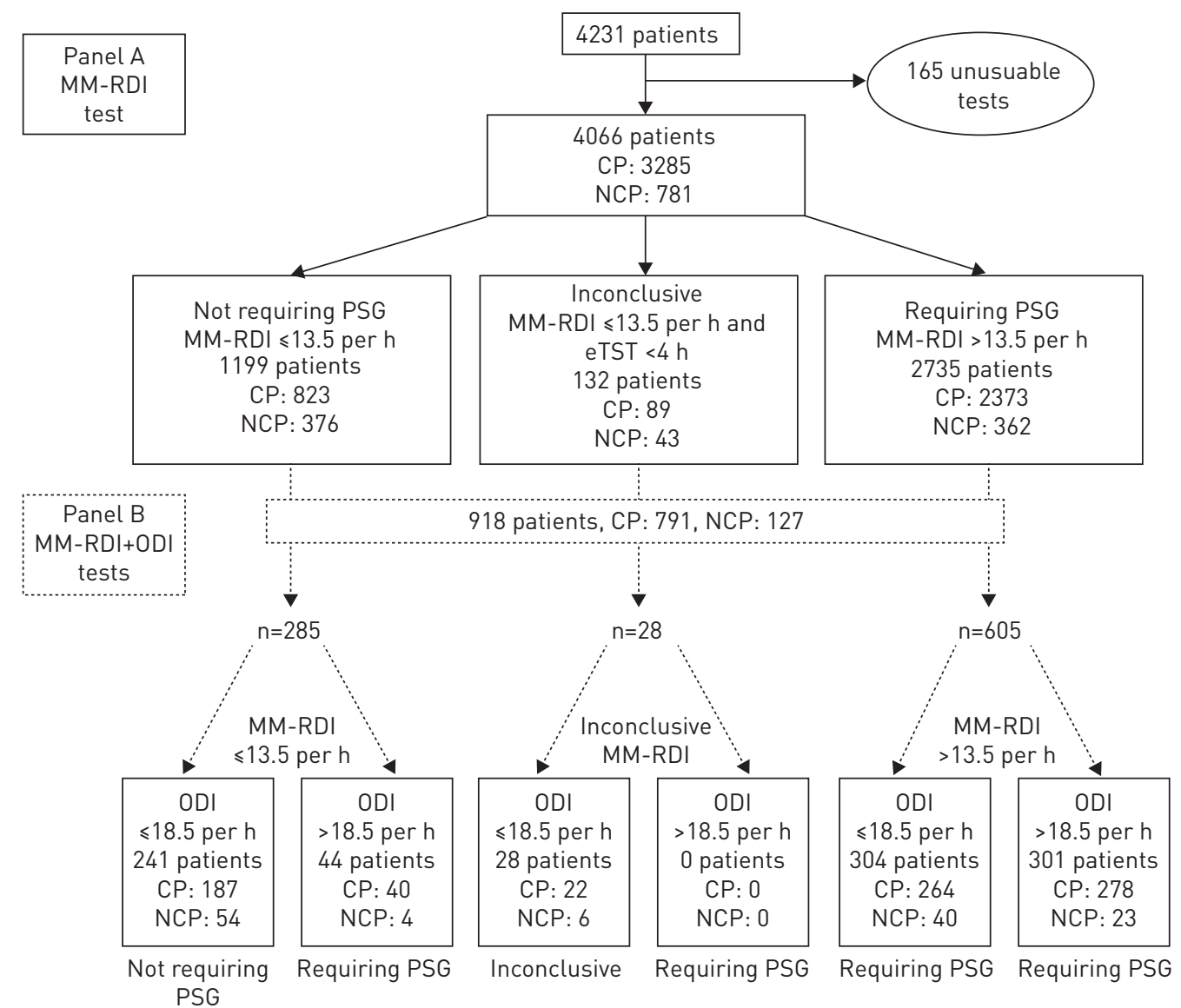

FIGURE 1 Flow chart demonstrating (in panel A) the total number of patients who performed a mandibular movement respiratory disturbance index (MM-RDI) test, the number of unusable tests and the final number of patients entering analysis. The latter were classified into three groups: patients classified as "not requiring polysomnography (PSG)" (left) with an MM-RDI $\leqslant 13.5$ per $\mathrm{h}$ and an estimated total sleep time (eTST) of at least $4 \mathrm{~h}$; patients classified as having "inconclusive" tests (centre) with an MM-RDI $\leqslant 13.5 \mathrm{per} \mathrm{h}$ and an eTST $<4 \mathrm{~h}$; and patients classified as "requiring PSG" (right) with an MM-RDI >13.5 per h. Inconclusive MM-RDI tests should be repeated. A significantly higher percentage of patients "requiring PSG" was found among those who had cardiologist prescribers (CP) versus those who had non-cardiologist prescribers (NCP) (Chi-squared test, $p<0.001$ ). Panel $B$ extends the flow chart to the 918 patients in panel $A$ who had simultaneous oximetry in addition to mandibular movement tracking. The three groups in panel $A$ are thus further subdivided, according to a $3 \%$ oxygen desaturation index (ODI) > or $\leqslant 18.5$ per $\mathrm{h}$ (requiring and not requiring PSG, respectively). In this panel, the final classification "requiring PSG" is conditioned by a MM-RDI $>13.5$ per $\mathrm{h}$ and/or an ODI >18.5 per $\mathrm{h}$. Oximetry provided additive diagnostic value in favour of the need for PSG among patients with an initially negative MM-RDI in $17.6 \%$ and $6.9 \%$ of CP and NCP patients, respectively (Chi-squared test, $p=0.069$ ).

Among the 4066 usable patient data-points, $67.3 \%$ and $29.5 \%$ were classified as 'requiring' and 'not requiring' PSG, respectively. Only a small percentage (3.25\%) of cases were classified as 'inconclusive' because of a MM-RDI $\leqslant 13.5$ per h, combined with less than $4 \mathrm{~h}$ of eTST (figure 1, panel A). These preliminary results differed further, according to the type of prescriber; $25 \%$ of those patients whose prescribing practitioner was a cardiologist (panel A) were classified as 'not requiring' PSG versus $48.14 \%$ (Chi-squared test, $\mathrm{p}<0.001$ ) of patients under the care of other types of prescribers (panel A).

We performed a complementary analysis in the subgroup of 918 patients who had a MM-RDI test with simultaneous oximetry incorporated into the MM magnetic sensor device. We used an oxygen desaturation index (ODI) cut-off at $>18.5$ per h (oxygen desaturation $>3 \%$ ), a threshold demonstrated to be associated with a PSG apnoea hypopnoea index (AHI) $>15$ per h, with a sensibility of $85.1 \%$ and specificity of $85.3 \%$ in a patient population from a previous sleep heart health study [8]. Oximetry provided additive diagnostic value in favour of the need for PSG among initially negative MM-RDI patients for $17.6 \%$ and $6.9 \%$ of the CP and NCP groups, respectively (Chi-squared test, $\mathrm{p}=0.069$ ). On the other hand, $48.7 \%$ of CP patients and $63.4 \%$ of NCP patients with a positive MM-RDI test had a negative ODI (Chi-squared test, $\mathrm{p}=0.026$; see figure 1, panel B). Furthermore, 53\%, 55\% and $40 \%$ of the whole, 
$\mathrm{CP}$ and non-CP populations, respectively, who had an ODI $\leqslant 18.5$ per $\mathrm{h}$ (that was not in favour of the need for PSG), had a value of MM-RDI >13.5 per h, in favour of PSG.

In order to fully compare the results of MM-RDI and ODI, and in particular, the manner in which the addition of the MM test to oximetry, or oximetry to the MM test enhances the need for PSG, we generated receiver operating curves (ROC) for the whole, $\mathrm{CP}$ and non-CP populations. Concerning the latter populations, the areas under the ROC for MM-RDI with a need for PSG, with the ODI pre-specified cut-off $>18.5$ per h, were 0.78 (95\% CI 0.75-0.81), 0.77 (95\% CI 0.74-0.81) and 0.79 (95\% CI 0.68-0.9), respectively. The areas under the ROC for the ODI with a need for PSG with the MM-RDI pre-specified cut-off $>13.5$ per h were 0.77 (95\% CI 0.74-0.80), 0.77 (95\% CI 0.74-0.8) and 0.77 (95\% CI 0.69-0.85), respectively. These ROC analyses support the coupling of MM-RDI and oximetry data when screening for PSG candidates.

When considering the feasibility and design of future studies, a screening test for SAS needs to be associated with a low rate of unusable test results. In the model developed by PIETZSCH et al. [5], a technical failure rate of $9 \%$ (range $7-12 \%$ ) was used. In this preliminary evaluation of MM-RDI as a screening test, we observed a technical failure rate of $3.9 \%$ and an inconclusive test rate of $3.2 \%$, suggesting that this parameter does not pre-empt future MM-RDI studies. In addition, MARTINOT et al. [7] reported that MM-RDI is associated with a positive likelihood ratio of 8.46 and a sensitivity of $89 \%$ for an RDI of 15 per $\mathrm{h}$, demonstrating concordance with evaluation criteria for obstructive sleep apnoea devices, as reviewed by ColLop et al. [9].

The question of whether or not to perform systematic patient screening is an unresolved issue, particularly in cardiac patients, even if they have no sleep-related symptoms [10-13]. Of course, the cost of diagnosis is one of the key points of the debate and cost-sparing procedures need to be developed. In this regard, the estimated cost of SAS diagnosis in 2016 was reportedly \$229 million in the US [14]. In 2012, the Haute Autorité de la Santé reported that the reimbursed cost for sleep disorder diagnosis in France was $€ 31.8$ million [15]. Considering our preliminary data, the economic gain would be around $25 \%$ of these diagnostic costs for cardiac patients and around $48.1 \%$ for non-cardiac patients. In contrast, the cost of the $\mathrm{MM}$ test for each patient is less than $€ 2.20$ (\$2.61), corresponding to the price of the magnetic sensor and its renewal, after every 50 patients. Coupled with oximetry, the cost is raised to $€ 0.3$ (\$0.36), corresponding to the utilisation of the same sensor during the associated warranty period. Given ambulatory conditions and automation, only material deployment and final interpretations represent further costs in addition to the $€ 2.5$ for necessary material.

This preliminary study has several limitations, the main one being that further clinical data and PSG results were unavailable, making it impossible to study other factors that might help to select PSG candidates. Although this study is preliminary in nature, it confirms that MM-RDI has potential as a simple screening tool for PSG candidates. Our results further suggest the synergy between oximetry and the MM-RDI test. The utility of MM-RDI, in addition to screening questionnaires and portable polygraphy, also requires further investigation. Even more important, by increasing the pre-test probability of SAS through the incorporation of MM-RDI tests, empirical continuous positive airway pressure (CPAP) treatment could become a more cost-effective strategy than the actual gold-standard of a full-night PSG with CPAP titration [5].

Marie-Caroline Rotty ${ }^{1,2}$, Jean-Pierre Mallet ${ }^{2,3}$, Jean-Christian Borel $^{4}$, Carey M. Suehs $\oplus^{3,5}$, Arnaud Bourdin ${ }^{2,3,6}$, Nicolas Molinari ${ }^{1,2,5}$ and Dany Jaffuel ${ }^{2,3,7}$

${ }^{1}$ Institut Montpelliérain Alexander Grothendieck, CNRS, Université de Montpellier, Montpellier, France. ${ }^{2}$ Association pour l'Assistance et la Réhabilitation à Domicile (APARD), Montpellier, France. ${ }^{3}$ Dept of Respiratory Diseases, Montpellier University Hospital, Hôpital Arnaud de Villeneuve, Montpellier, France. ${ }^{4}$ AGIR à dom, Meylan, France.

${ }^{5}$ Dept of Medical Information, Montpellier University Hospital, Hôpital La Colombière, Montpellier, France.

${ }^{6}$ PhyMedExp (INSERM U 1046, CNRS UMR9214), Université de Montpellier, Montpellier, France. ${ }^{7}$ Pulmonary Disorders and Respiratory Sleep Disorders Unit, Polyclinic Saint-Privat, Boujan sur Libron, France.

Correspondence: Dany Jaffuel, Dept of Respiratory Diseases, CHRU Montpellier, 371, Avenue Doyen Giraud, 34295 Montpellier Cedex 5, France. E-mail: dany.jaffuel@wanadoo.fr

Received: May 272017 | Accepted after revision: Sept 062017

Clinical trial: This study is registered at clinicaltrials.gov with identifier number NCT03129984.

Conflict of interest: None declared.

Acknowledgements: Data were provided by the Brizzy registry (Nomics, Belgium). However, Nomics was in no way involved in this analysis, nor did they provide any kind of incentive to any of the authors. 


\section{References}

1 Heinzer R, Marti-Soler H, Haba-Rubio J. Prevalence of sleep apnoea syndrome in the middle to old age general population. Lancet Respir Med 2016; 4: e5-e6.

2 Sharples LD, Clutterbuck-James AL, Glover MJ, et al. Meta-analysis of randomised controlled trials of oral mandibular advancement devices and continuous positive airway pressure for obstructive sleep apnoea-hypopnoea. Sleep Med Rev 2016; 27: 108-124.

3 Arzt M, Hetzenecker A, Lévy P. Obstructive sleep apnoea in acute coronary syndrome: the invisible threat? Eur Respir J 2017; 49: 1602539.

4 Flemons WW, Douglas NJ, Kuna ST, et al. Access to diagnosis and treatment of patients with suspected sleep apnea. Am J Respir Crit Care Med 2004; 169: 668-672.

5 Pietzsch JB, Garner A, Cipriano LE, et al. An integrated health-economic analysis of diagnostic and therapeutic strategies in the treatment of moderate-to-severe obstructive sleep apnea. Sleep 2011; 34: 695-709.

6 Gray EL, Barnes DJ. Beyond the thermistor: novel technology for the ambulatory diagnosis of obstructive sleep apnoea. Respirology 2017; 22: 418-419.

7 Martinot J-B, Borel J-C, Cuthbert V, et al. Mandibular position and movements: suitability for diagnosis of sleep apnoea. Respirology 2017; 22: 567-574.

8 Schlotthauer G, Di Persia LE, Larrateguy LD, et al. Screening of obstructive sleep apnea with empirical mode decomposition of pulse oximetry. Med Eng Phys 2014; 36: 1074-1080.

9 Collop NA, Tracy SL, Kapur V, et al. Obstructive sleep apnea devices for out-of-center (OOC) testing: technology evaluation. J Clin Sleep Med 2011; 7: 531-548.

10 US Preventive Services Task Force, Bibbins-Domingo K, Grossman DC, et al. Screening for obstructive sleep apnea in adults: US preventive services task force recommendation statement. JAMA 2017; 317: 407-414.

11 Aurora RN, Quan SF. Quality measure for screening for adult obstructive sleep apnea by primary care physicians. J Clin Sleep Med 2016; 12: 1185-1187.

12 Epstein LJ, Kristo D, Strollo PJ, et al. Clinical guideline for the evaluation, management and long-term care of obstructive sleep apnea in adults. J Clin Sleep Med 2009; 5: 263-276.

13 de Batlle J, Turino C, Sánchez-de-la-Torre A, et al. Predictors of obstructive sleep apnoea in patients admitted for acute coronary syndrome. Eur Respir J 2017; 49: 1600550.

14 American Academy of Sleep Medicine. Hidden health crisis costing America billions: underdiagnosing and undertreating obstructive sleep apnea draining healthcare system. Darien, American Academy of Sleep Medicine, 2016. https://aasm.org/economic-burden-of-undiagnosed-sleep-apnea-in-u-s-is-nearly-150b-per-year/ Date last accessed: October 31, 2017. Date last updated: August 08, 2016.

15 Haute Autorité de santé. Place et conditions de réalisation de la polysomnographie et de la polygraphie respiratoire dans les troubles du sommeil - Rapport d'évaluation [The place of and conditions for carrying out polysomnography and respiratory polygraphy in sleep disorders - Brief]. www.has-sante.fr/portail/jcms/c_1056842/fr/place-etconditions-de-realisation-de-la-polysomnographie-et-de-la-polygraphie-respiratoire-dans-les-troubles-du-sommeil-rapportd-evaluation Date last accessed: October 31, 2017. Date last updated: May 2012. 